\title{
Carcinosarcoma of the esophagus: A report of 6 cases associated with zinc finger E-box-binding homeobox 1 expression
}

\author{
HIROKI HARADA ${ }^{1}$, KEI HOSODA ${ }^{1}$, HIROMITSU MORIYA ${ }^{1}$, HIROAKI MIENO ${ }^{1}$, AKIRA EMA ${ }^{1}$, MARIE WASHIO ${ }^{1}$, \\ MARIKO KIKUCHI $^{2}$, YOSHIMASA KOSAKA ${ }^{2}$, MASAHIKO WATANABE $^{1}$ and KEISHI YAMASHITA ${ }^{1,3}$ \\ Departments of ${ }^{1}$ Surgery and ${ }^{2}$ Breast and Endocrine Surgery; ${ }^{3}$ Division of Advanced Surgical Oncology, \\ Department of Research and Development Center for New Medical Frontiers, Kitasato University School of Medicine, \\ Sagamihara, Kanagawa 252-0375, Japan
}

Received August 11, 2017; Accepted September 6, 2018

DOI: $10.3892 / \mathrm{ol} .2018 .9585$

\begin{abstract}
Esophageal carcinosarcoma (ECS) has been suggested to result from an epithelial mesenchymal transition (EMT) phenomenon. However, knowledge on its underlying molecular features is limited. The clinical and pathological features, and the prognosis of ECS require further investigation. In the present study, a total of 325 patients with esophageal tumors were observed between January 2004 and December 2014, of which 6 patients were diagnosed pathologically with ECS. The clinicopathological features were compared with those of corresponding cases with the identical pathological $\mathrm{T}$ stage (pT) of esophageal squamous cell carcinoma (ESCC). In terms of the clinical $\mathrm{T}$ stage (cT), the 6 cases were composed of cT1, cT2, cT3 and cT4 in 1, 1, 3 and 1 case, respectively. Nevertheless, pT was eventually diagnosed as pT1 in all cases. There was a large discrepancy between clinically diagnosed depth of tumor invasion prior to surgery and depth of tumor invasion following surgery. Zinc finger E-box-binding homeobox 1 (ZEB1), an EMT-associated transcription factor, was expressed only in the sarcoma component in all 6 cases of ECS. The ECS cases had a significantly poorer prognosis compared with the 115 pT1 ESCC cases. The present study suggests that the depth of invasion of ECS lesions does not correspond with their respective size, and the EMT of the carcinoma component may affect the prognosis by overexpression of the ZEB1 gene.
\end{abstract}

\section{Introduction}

Esophageal carcinosarcoma (ECS) is a rare type of esophageal cancer that was designated as such by Virchow et al (1) due to

Correspondence to: Professor Masahiko Watanabe, Department of Surgery, Kitasato University School of Medicine, 1-15-1 Kitasato, Minami, Sagamihara, Kanagawa 252-0375, Japan

E-mail: intl-aff@kitasato-u.ac.jp

Key words: esophageal carcinosarcoma, epithelial mesenchymal transition, zinc finger E-box-binding homeobox 1 expression, esophageal squamous cell carcinoma the presence of carcinomatous and sarcomatous components. The components consist of spindles or polymorphous tumor cells with a mesenchymal character. This tumor often presents with polypoid growth with a stalk, and is characterized by the presence of esophageal squamous cell carcinoma (ESCC) in situ surrounding the stalk (2). It has been suggested that the sarcoma component is derived from SCC (3-5).

Epithelial-mesenchymal transition (EMT) has been reported as an important means of tumor invasion and metastasis in numerous types of canonical cancer $(6,7)$. Zinc finger E-box-binding homeobox 1 (ZEB1) is associated with EMT transcription factors (8). Members of the microRNA (miR)-200 family have been reported to serve an important role in dysregulating the epithelial phenotype by targeting ZEB factors, thereby preventing E-cadherin downregulation and resulting in EMT (9). In human cancer, ZEB1 expression has been indicated to be increased when miR-200 expression is decreased due to promoter DNA methylation $(10,11)$. The EMT activator ZEB1, in particular, has also been indicated to confer stemness and resistance to anticancer treatment (9). ZEB1 was specifically recognized in the sarcoma component compared with the carcinoma component in spindle cell carcinoma of the esophagus, among which a number of ECS cases were included (8). To the best of our knowledge, information regarding ZEB1 expression in ECS is limited.

In the present study, the aim was to clarify the molecular features associated with prognosis by comparing the clinicopathological factors and prognosis of ECS and ESCC in pT1.

\section{Materials and methods}

Patients and data collection. Esophagectomy was performed in 325 patients with esophageal tumors at Kitasato University Hospital (Sagamihara, Japan) between January 2004 and December 2014, and 5 male and 1 female patient (1.8\%), with a mean age of 68 years (range, 60-77 years), were diagnosed with ECS. The present study was conducted in accordance with the Declaration of Helsinki and approved by the Research Ethics Committee of Kitasato University School of Medicine (Sagamihara, Japan). Written consent was obtained from all patients. All tissue samples were collected at the Kitasato University Hospital. The clinical and pathological features 
were analyzed and tumor stage was classified according to the 6th edition of the Union for International Cancer Control Tumor-Node-Metastasis (UICC-TNM 6th edition) staging system of esophageal cancer (12). The median follow-up time was 29 months (range, 16-33 months).

Immunohistochemical staining. Serial tissues sections (4- $\mu \mathrm{m}$ thick) were fixed in $10 \%$ formalin at room temperature for 16-24 $\mathrm{h}$ and embedded in paraffin. The slices were incubated with $3 \% \mathrm{H}_{2} \mathrm{O}_{2}$ at room temperature for $5 \mathrm{~min}$ to deactivate endogenous peroxidase and subsequently washed with PBS. Rabbit anti-human ZEB1 polyclonal antibody (dilution 1:100; cat. no. HPA027524; Atlas Antibodies AB, Stockholm, Sweden), rabbit Snail 1 polyclonal antibody (dilution, 1:500; cat. no. GTX125918; Gene Tex, Los Angeles, USA) and rabbit Twist-related protein 1 (Twist 1) polyclonal antibody (dilution, 1:500; cat. no. ab50581; Abcam, Cambridge, UK) were added, and the slices were incubated at $4^{\circ} \mathrm{C}$ overnight. Immune complexes were amplified using a Vectastain Universal Elite ABC kit (dilution, 1:50; cat. no. PK-6200; Vector Laboratories, Inc., Burlingame, CA, USA), at room temperature for $10 \mathrm{~min}$, according to the manufacturer's protocols. These complexes were subsequently detected by incubation at room temperature with the chromogen 3,3'-diaminobenzidine (3\%) for $1 \mathrm{~min}$. The invasive front of the carcinoma components and the sarcomatous areas were compared. Using a light microscope (Olympus AX80; Olympus Corporation, Tokyo, Japan) (x20 magnification), positive staining was defined as nuclear immunoreactivity in neoplastic cells. Hematoxylin at $0.1 \%$ concentration was used to stain at room temperature for $5 \mathrm{~min}$, then $0.025 \%$ eosin was used to stain at room temperature for $3 \mathrm{~min}$. As a control tissue of ZEB1 in the present study, according to the study by Kikuchi et al (13), mammary gland tissues were also stained. Triple-negative breast cancer tissues demonstrating the strongest staining were used as a positive control, whereas normal breast tissues that did exhibit staining were used as a negative control (13). The positive and negative controls were primary tumors and the corresponding non-cancerous tissues from 3 patients with triple-negative invasive breast cancer who all underwent a partial resection of the breast in September 1999 at Kitasato University Hospital. Based on previous studies (14-17), gastric adenocarcinoma tissues with confirmed staining were used as a positive control for Twist 1 and Snail 1, while a non-cancerous mucosa sample was used as the negative control. The positive and negative controls were primary tumors and the corresponding non-cancerous tissues from 3 patients with macroscopic type 0-IIc early gastric cancer who underwent laparoscopic gastrectomy in October and November, 2016, and November 2005, respectively, at Kitasato University Hospital.

Statistical analysis. All statistical analyses were performed using JMP ${ }^{\circledR} 11.0$ software (SAS Institute Inc., Cary, NC, USA). Frequency tables were analyzed using the $\chi^{2}$ test, while the significance of categorical variables was evaluated with the likelihood ratio. Disease-specific survival was measured from the date of diagnosis to the time of patient mortality due to a specific disease, or censored at the date of the last follow-up evaluation. For evaluation of relapse-free survival, recurrence was defined as development of local recurrence, distant metastasis or patient mortality from ECS (whichever occurred first). Survival functions were estimated by life tables and the Kaplan-Meier method, and compared by log-rank test. $\mathrm{P}<0.05$ was considered to indicate a statistically significant difference.

\section{Results}

Patient characteristics. The characteristics of the patients with ECS are indicated in Table I. The mean age was 68 years. With the exception of 1 unknown case, all patients had a history of smoking and drinking. The main symptoms reported were difficulty in swallowing, coughing and chest pain in 3, 2 and 1 case, respectively. All cases had an American Society of Anesthesiologists-Physical Status of $\leq 2$ (18). All patients underwent computed tomography, and 5 patients underwent upper gastrointestinal contrast studies. Regarding clinical T stage (cT), cT1, cT2, cT3 and cT4 were indicated in 1, 1, 3 and 1 case, respectively (Fig. 1). The longitudinal length of the tumor was $2.5-10.5 \mathrm{~cm}$ (mean, $6.5 \mathrm{~cm}$ ). Pathological T stage (pT) was eventually diagnosed as pT1 in all cases. According to Japanese Classification of Esophageal Cancer 11th Edition (2), the final pathological morphological type was pedunculated type, sessile (broad based) type, and pedunculated and slightly elevated type in 4, 1, and 1 cases, respectively. All patients underwent radical surgery with complete tumor resection.

Patient 1 received preoperative chemoradiotherapy (CRT), where radiotherapy was applied at $40 \mathrm{~Gy}$ due to suspected tracheal invasion (cT4). The CRT therapy included radiotherapy concurrent with nedaplatin +5 -fluorouracil (FU) chemotherapy due to renal dysfunction. Nedaplatin $+5-\mathrm{FU}$ chemotherapy consisted of two courses of chemotherapy with 5-fluorouracil (800 $\mathrm{mg} / \mathrm{m}^{2}$ on days $\left.1-5\right)$ and nedaplatin (90 mg/m² on day 1 ) every 4 weeks. Postoperative adjuvant CRT was performed in patient 2 due to vigorous vascular invasion and lymph node metastasis in the pathological results, and chemotherapy [nedaplatin $\left(50 \mathrm{mg} / \mathrm{m}^{2}\right.$ on day 1) +5 -FU (400 mg/m² on days $1-5)$ ] was additionally used due to renal dysfunction. The pathological depth of invasion was pT1 in all cases, and there was a large discrepancy between clinically diagnosed depth of tumor invasion prior to surgery and depth of tumor invasion following surgery. Lymph node metastasis was indicated in 2 cases. Postoperative complications included hoarseness due to recurrent laryngeal nerve paralysis in 3 cases, and anastomotic leakage [Clavien-Dindo classification (19) grade IIIa], which caused a gastrointestinal tract to lung fistula, in 1 case. Postoperative recurrence was observed in 3 cases despite the superficial depth of invasion in all cases. The 3 patients with recurrence succumbed at 20 , 28 , and 32 months due to the progression of recurrence in the lymph nodes, liver and lungs/bones, respectively. Patient 2 , in particular, presented with multiple lung metastases and fifth rib metastasis at 16 months post-surgery, despite preoperative adjuvant CRT. Despite administration of 8 courses of cisplatin $\left(15 \mathrm{mg} / \mathrm{m}^{2}\right.$ on day 1$)+5-\mathrm{FU}\left(750 \mathrm{mg} / \mathrm{m}^{2}\right.$ on days 1-5) and 1 course of cisplatin $\left(90 \mathrm{mg} / \mathrm{m}^{2}\right.$ on day 1$)$ + irinotecan $\left(75 \mathrm{mg} / \mathrm{m}^{2}\right.$ on day 1$)$, the patient succumbed at 32 months post-surgery. Patient 4 presented with ECS with pT1 (UICC-TNM 6th edition) lymph node metastasis 0 , lymphatic invasion 0 , venous invasion 0 and granulocyte 
Table I. Characteristic of patients with esophageal carcinoma.

\begin{tabular}{|c|c|c|c|c|c|c|}
\hline Variables & Case 1 & Case 2 & Case 3 & Case 4 & Case 5 & Case 6 \\
\hline Sex & Male & Female & Male & Male & Male & Male \\
\hline Age, years & 61 & 60 & 73 & 77 & 68 & 71 \\
\hline Smoking status & Former & Unknown & Current & Current & Current & Former \\
\hline Chief complaint & Chest pain & Dysphagia & Cough & Cough & Dysphagia & Dysphagia \\
\hline Location & Mt & Mt & $\mathrm{Lt}$ & $\mathrm{Ut}$ & Mt & Mt \\
\hline Clinical T factor & cT4 & cT3 & cT3 & cT3 & cT2 & $\mathrm{cT} 1$ \\
\hline Albumin level, g/dl & 4.1 & 4 & 3.3 & 3.3 & 3.4 & 3.4 \\
\hline Neutrophil to lymphocyte ratio & 3 & 2.9 & 1.7 & 2.4 & 6.9 & 6.6 \\
\hline ASA-PS & Class 1 & Class 1 & Class 2 & Class 1 & Class 1 & Class 2 \\
\hline Tumor length, cm & 2.5 & 10.5 & 4.5 & 6.5 & 8.5 & 6.6 \\
\hline Macroscopic type & 0-Ip & 0-Ip & 0 -Ip & 0 -Isp & 0-Ip+IIa & 0-Ip \\
\hline Lymphatic invasion & Unknown & 2 & 0 & 0 & 1 & 0 \\
\hline Venous invasion & Unknown & 2 & 0 & 0 & 1 & 0 \\
\hline pT factor & pT1 & pT1 & pT1 & pT1 & pT1 & pT1 \\
\hline $\mathrm{pN}$ factor & pNO & pN1 & $\mathrm{pNO}$ & pN0 & $\mathrm{pN} 1$ & pNO \\
\hline Immunohistochemical findings & Unknown & Vimentin(+) & Vimentin(+) & $\mathrm{G}-\mathrm{CSF}(+)$ & Vimentin(+) & Vimentin(+) \\
\hline Initial treatment & $\begin{array}{c}\mathrm{NF}+\mathrm{RT}+ \\
\text { Resection }\end{array}$ & $\begin{array}{c}\text { Resection + } \\
\mathrm{NF}+\mathrm{RT}\end{array}$ & Resection & Resection & Resection & Resection \\
\hline Tumor recurrence & No & Yes & No & Yes & Yes & No \\
\hline Treatment following recurrence & None & $\begin{array}{c}\text { 5-FU + cisplatin } \\
\text { irinotecan }+ \\
\text { cisplatin }\end{array}$ & None & $5-\mathrm{FU}+$ & $\begin{array}{c}\text { BSC } \\
\text { cisplatin }\end{array}$ & None \\
\hline Prognosis & Alive & $\begin{array}{l}\text { Succumbed } \\
\text { to tumor }\end{array}$ & $\begin{array}{l}\text { Succumbed } \\
\text { to AMI }\end{array}$ & $\begin{array}{c}\text { Succumbed } \\
\text { to tumor }\end{array}$ & $\begin{array}{l}\text { Succumbed } \\
\text { to tumor }\end{array}$ & Alive \\
\hline
\end{tabular}

Mt, middle thoracic esophagus; Lt, Lower thoracic esophagus; Ut, Upper thoracic esophagus; ASA-PS, American Society of Anesthesiologists physical status; 5-FU, fluorouracil; NF, nedaplatin + 5-FU; RT, radiationtherapy; AMI, acute myocardial infarction; G-CSF, granulocyte colony-stimulating factor; BSC, best supportive care; pT, pathological depth of tumor invasion; pN, pathological lymph node stage.

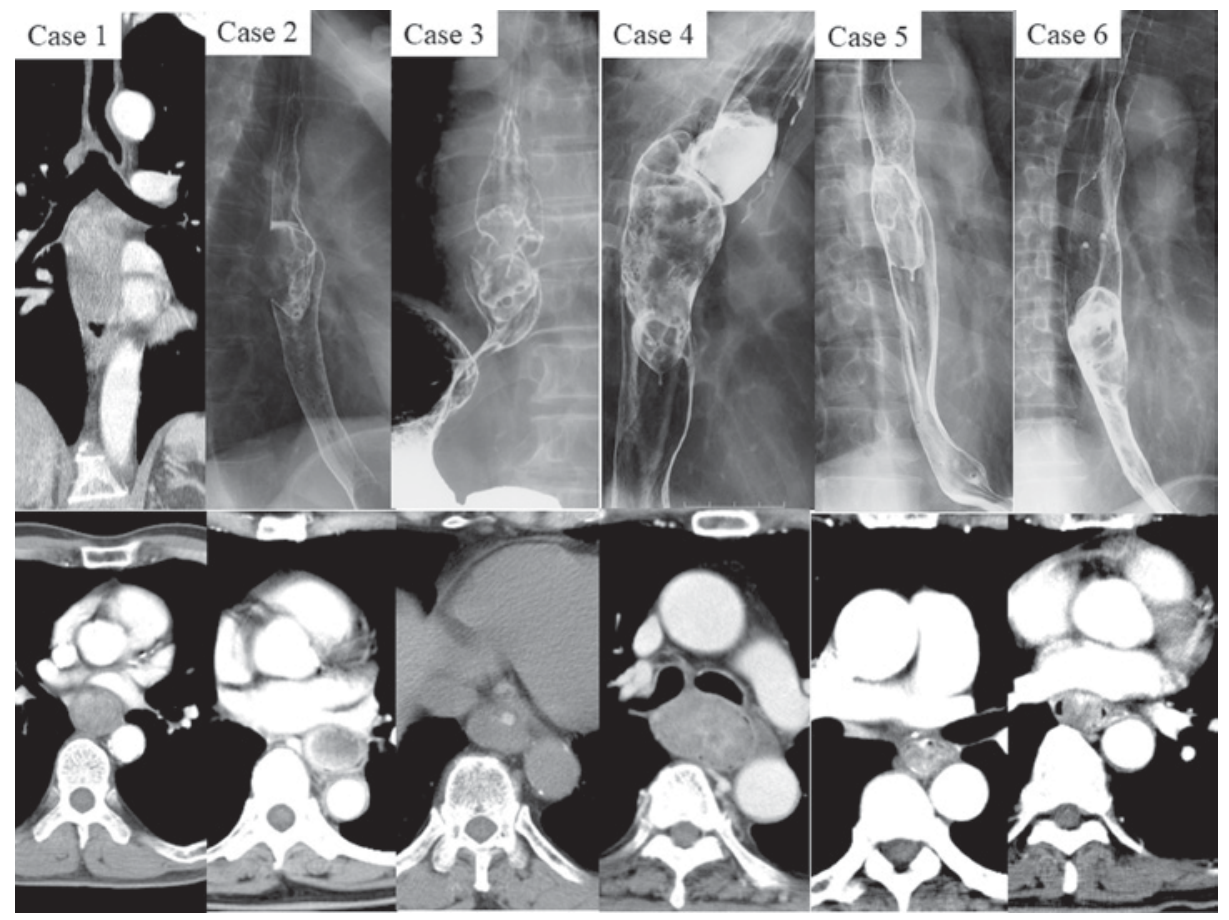

Figure 1. Radiography of the upper gastrointestinal tract and chest computed tomography indicating an intraluminal polypoid lesion in all 6 cases. The mean tumor size was $6.5 \mathrm{~cm}$ (major axis, $2.5-10.5 \mathrm{~cm}$ ). 


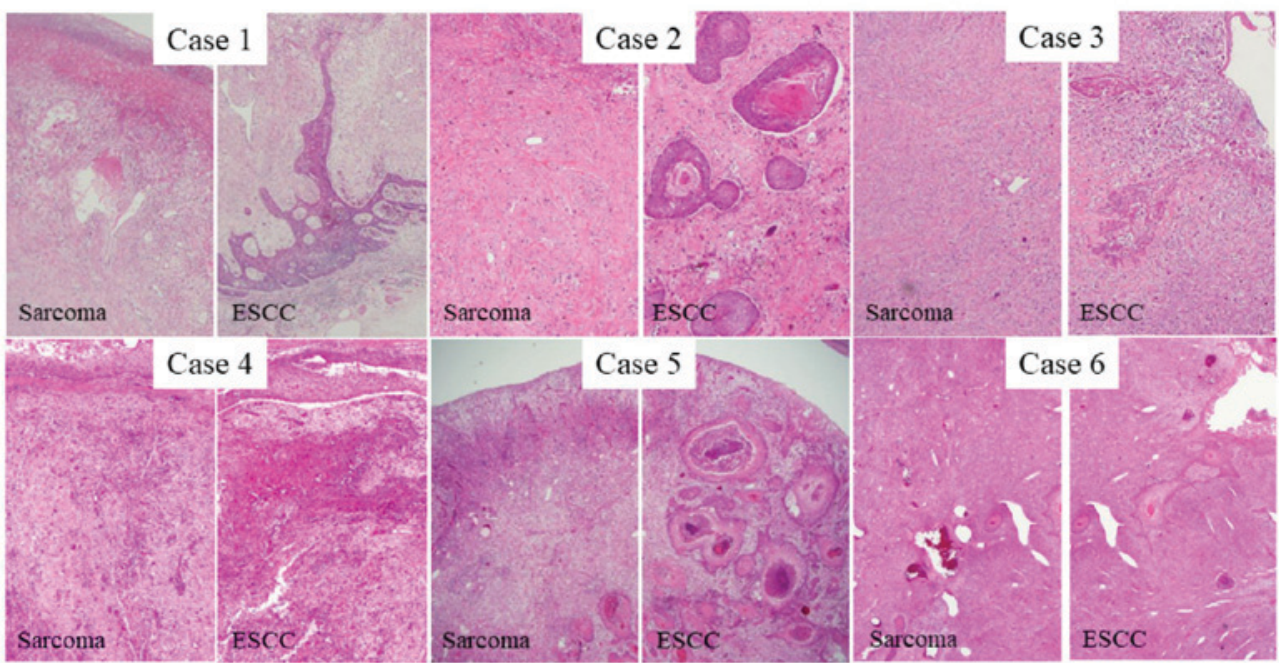

Figure 2. Microscopic appearance of squamous cell carcinoma and spindle-shaped tumor cells of the carcinosarcoma component in all 6 cases (hematoxylin and eosin stain, x20 magnification). ESCC, esophageal squamous cell carcinoma.

colony-stimulation factor-positive tumor cells in the immunohistochemical analysis. This patient experienced recurrence of lymph node metastasis at 6 months post-surgery, and despite administration of 8 courses of cisplatin $\left(60 \mathrm{mg} / \mathrm{m}^{2}\right.$ on day 1$)+5-\mathrm{FU}\left(800 \mathrm{mg} / \mathrm{m}^{2}\right.$ on days $\left.1-5\right)$, the patient additionally developed brain metastasis and succumbed at 20 months post-surgery. Patient 5 presented with liver metastasis at 23 months post-surgery and additionally developed pleural dissemination, lung metastasis and para-aortic lymph node metastasis at 26 months post-surgery. The patient succumbed 2 months later.

Pathological features. Histopathological findings indicated carcinomatous cells and sarcomatous cells (Fig. 2). The sarcomatous component exhibited spindle-shaped heterotypic cells with varying sizes of enlarged nuclei. ESCC components, scattered in an island shape, were indicated in the lesion. The two components were separate in certain places and intermingled in others. All cases exhibited ZEB1 expression in neoplastic cells distributed uniformly in the sarcomatous component (Fig. 3). Neoplastic cells in the carcinoma components were negative for ZEB1. In 2 cases, metastatic lymph nodes were negative for ZEB1 (Fig. 4). The expression of Twist 1 and Snail 1, which are EMT-associated factors similar to ZEB1, was confirmed. As a result, Snail 1 was weakly expressed in the sarcoma component and the ESCC component (Fig. 5). Twist 1 was strongly expressed in the sarcoma and the ESCC component (Fig. 6). There was no indication that Twist 1 and Snail 1 were expressed specifically in the sarcoma component.

Treatment and clinical outcome. Prognostic analysis of 115 cases of pT1 ESCC with the same depth of tumor invasion as ECS (Table II) indicated a 5-year disease-specific survival rate of $40.0 \%$ in ECS and $90.6 \%$ in ESCC. ECS had a significantly worse prognosis compared with ESCC in pT1 cases $(\mathrm{P}=0.0016$; Fig. 7A). The 5-year relapse-free survival rate was $22.2 \%$ in ECS and $77.1 \%$ in ESCC, and ECS had a significantly worse prognosis, compared with ESCC ( $\mathrm{P}=0.0267$; Fig. 7B).

\section{Discussion}

In the present study, the clinicopathological features of ECS were examined. The results indicated that there was a large discrepancy between the cT factor and the pT factor in ECS. Even in cases with a predicted depth of invasion of cT2 or beyond with large polypoid tumors, the pT factor in all cases indicated that the depth of tumor was superficial invasion only. Regarding the fact that all cases were T1 cases, this was uncommon. According to previous studies, half of reported cases were pT1 (20-22). Due to the high level of patient awareness with regard to health in the area in which the Kitasato University Hospital is situated, the diagnosis of the disease at an early stage is more likely. However, as a number of patients harbored large tumors occupying the lumen, the present study reported an unexpectedly large discrepancy between the pathological depth of tumor invasion and the clinically predicted depth of invasion. It has been reported that $>90 \%$ of ECS lesions are pedunculated or semipedunculated (23). The polypoid lesions have a predominantly sarcoma component, whereas the superficial ESCC component is mainly present in the stem and base (2). Therefore, sarcomatous metaplasia originally occurs in superficial carcinoma, and only the sarcoma-like component increases rapidly toward the lumen (2).

It has been suggested that in carcinosarcoma, the sarcoma component may derive from EMT $(24,25)$. EMT is involved in a number of developmental milestones, including gastrulation, neural crest formation and heart morphogenesis, which rely on the plastic transition between the epithelium and mesenchyme (6). By contrast, during the progression of epithelial tumors, cancer cells develop increased motility and invasiveness (6). EMT has been established as an important step in the metastatic cascade of epithelial tumors (7). There are numerous molecules that could explain EMT, including ZEB1, Twist 1 and Snail; however, in esophageal carcinosarcoma, ZEB1 has been suggested to serve a critical role in the EMT process (7). In the present study, it is noteworthy that high expression of ZEB1 was recognized only in the sarcoma component. For this 


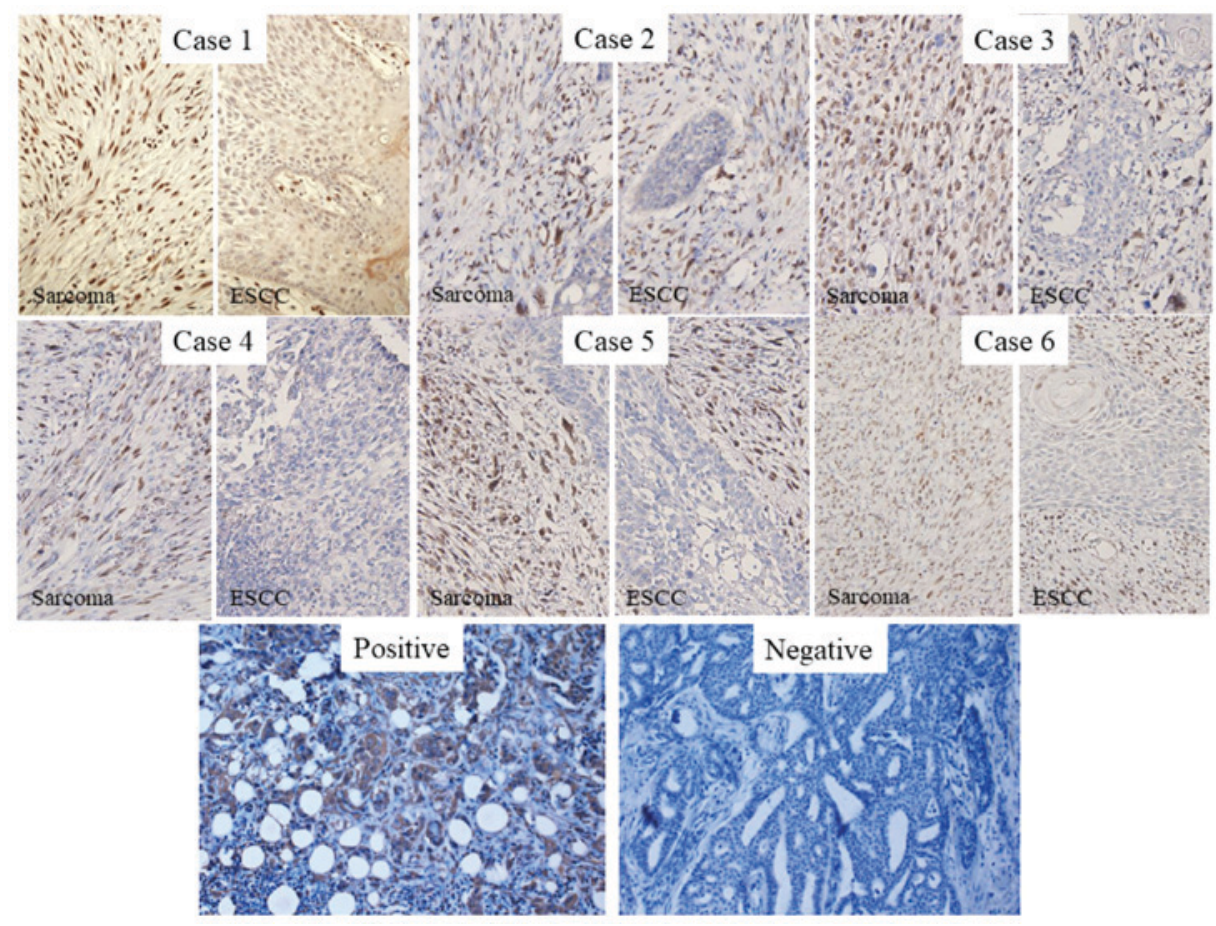

Figure 3.ZEB1 expression (magnification, x100) in neoplastic cells distributes uniformly in the sarcomatous component. All cases indicated the ZEB1 staining pattern. Normal breast tissues and triple-negative breast cancer tissue were defined as negative control and positive control, respectively. ESCC, esophageal squamous cell carcinoma; ZEB1, zinc finger E-box-binding homeobox 1 expression.

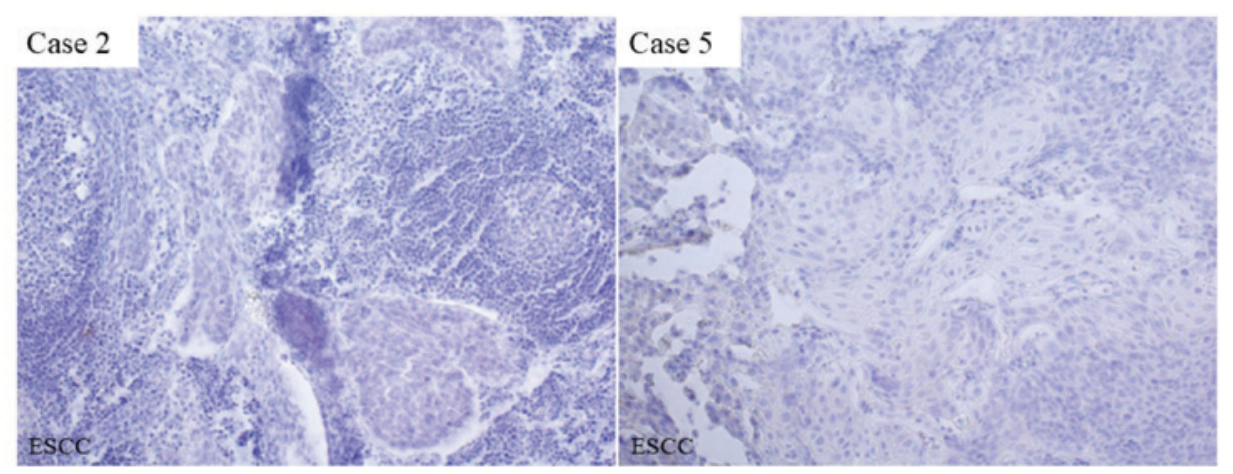

Figure 4. Metastatic lesion of SCC in the lymph node. Cases 2 and 5 were negative for the zinc finger E-box-binding homeobox 1 expression staining pattern. ESCC, esophageal squamous cell carcinoma.

reason, immunohistochemical staining was confirmed in all 6 cases, and as depicted in Fig. 3, the expression of ZEB1 was confirmed to be increased in the ECS component, confirm with the ESCC component. This result suggests that ZEB1 upregulation in ESCC cells may cause EMT and the transformation to carcinosarcoma. In addition, another study reported that phospholipid glutathione peroxidase (GPX4) inhibition, which induces ferroptic cell death in therapy-resistant cancer cells across diverse mesenchymal cell-state contexts, did not exhibit consistent sensitization in Snail 1 and Twist 1, but ZEB1 was correlated with mesenchymal state sensitivity to GPX4 inhibition (26). ZEB1 is a transcriptional factor that can induce EMT through critical mediators, including transforming growth factor $\beta 1(27,28)$ and miR-200 (29-31), suggesting that the clinical data of the present study may support the underlying molecular mechanism of ZEB1 in EMT.
Zhang et al (32) reported that spindle cell carcinoma (SpCC) had a depth of pathological T1/2 ( $<<0.001)$ and a good prognosis $(\mathrm{P}=0.044)$, compared with typical SCC, with increased tumor depth, compare with SpCC. In the present study, the prognosis of ECS was significantly worse compared with that of ESCC in terms of disease-specific survival rate $(\mathrm{P}=0.0016)$. The prognostic relevance between SpCC and ESCC differed between the study by Zhang et al (32) and the present study. However, in this previous study, the depth of tumor was significantly shallower in SpCC cases compared with that in typical SCC cases. It was considered that an identical depth of tumor may cancel out the prognostic difference observed. In fact, when the pathological factors of the patient background were stratified into pT1/2 and the prognostic analysis was performed, it was reported that there was no significant difference in the prognosis between SpCC and 


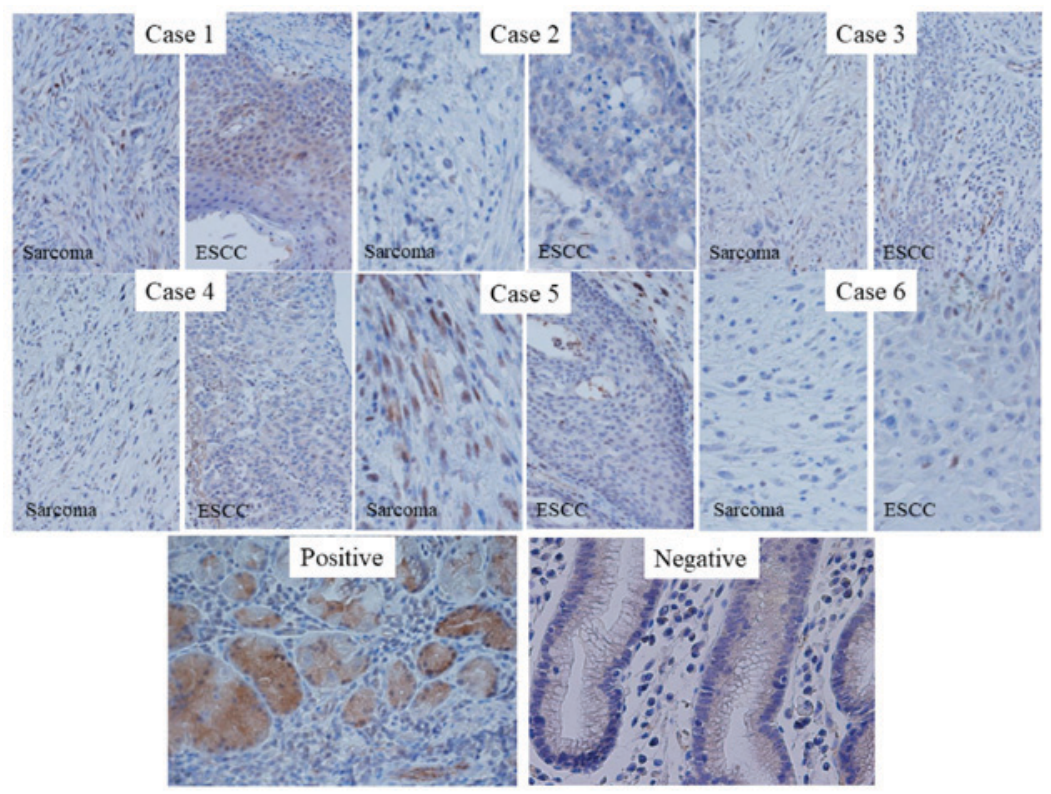

Figure 5. Sarcoma and carcinoma areas are weakly immunohistochemically positive for the expression of Snail 1 (magnification, $x 100)$. There was no indication that Snail 1 was expressed specifically in the sarcoma component. All cases indicated the Snail 1 staining pattern (antigen recognized by a rabbit Snail 1 polyclonal antibody). Non-cancerous adjacent mucosa and early gastric cancer tissues were defined as the negative control and positive control, respectively. Snail 1, snail family transcriptional repressor 1; ESCC, esophageal squamous cell carcinoma.

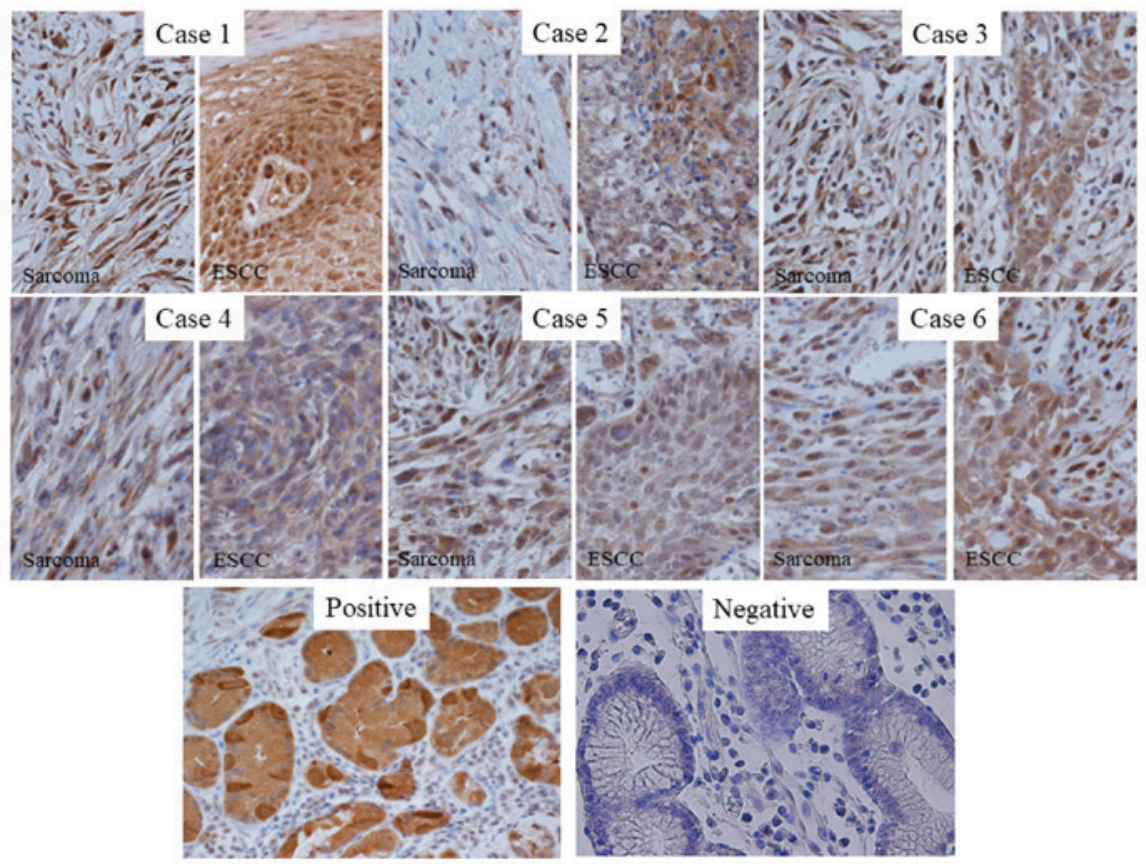

Figure 6. Sarcoma and carcinoma areas are strongly immunohistochemically positive for the expression of Twist 1 (magnification, x100). There was no indication that Twist 1 was expressed specifically in the sarcoma component. All cases indicated the Twist 1 staining pattern (antigen recognized by a rabbit Twist 1 polyclonal antibody). Non-cancerous adjacent mucosa and early gastric cancer tissue were defined as the negative control and positive control, respectively. Twist 1, Twist-related protein 1; ESCC, esophageal squamous cell carcinoma.

typical SCC (32). Therefore, the present study, to the best of our knowledge, included novel findings comparing ECS cases and ESCC cases with pT1.

It has been reported that the expression of ZEB1 in the sarcoma component may be a cause of poor prognosis by participating in treatment resistance, for example, against chemotherapy, and causing distant metastasis (33). In addition, previous studies reported that the inhibition of ZEB1 expression suppressed the tumorigenesis of breast cancer cells (34), that ZEB1 promoted metastasis and loss of cell polarity by repressing the expression of lethal giant larvae homolog 2 in colorectal cancer cells (35), and that ZEB1 was associated with the resistance to chemotherapeutic agents, including gemcitabine, 5-FU and cisplatin in pancreatic cancer cells (36). Recurrent cases were observed in the present study, despite administration of postoperative adjuvant therapy with 
Table II. Univariate analysis of esophageal carcinosarcoma and esophageal squamous cell carcinoma with the same depth of tumor invasion.

\begin{tabular}{|c|c|c|c|}
\hline Characteristics & pT1 carcinosarcoma $(n=6)$ & pT1 squamous cell carcinoma $(n=115)$ & P-value \\
\hline Sex & & & 0.85 \\
\hline Male & 5 & 99 & \\
\hline Female & 1 & 16 & \\
\hline Age, years & & & 0.65 \\
\hline$<65$ & 2 & 49 & \\
\hline$>64$ & 4 & 66 & \\
\hline Location of tumor & & & 0.99 \\
\hline Cervical esophagus & 0 & 1 & \\
\hline Upper thoracic esophagus & 1 & 20 & \\
\hline Middle thoracic esophagus & 4 & 67 & \\
\hline Lower thoracic esophagus & 1 & 24 & \\
\hline Abdominal esophagus & 0 & 3 & \\
\hline $\mathrm{pN}$ factor & & & 0.96 \\
\hline $\mathrm{pN} 1$ & 2 & 42 & \\
\hline pNO & 4 & 73 & \\
\hline Procedure of esophagectomy & & & 0.61 \\
\hline VATS & 1 & 80 & \\
\hline Thoracotomy & 5 & 35 & \\
\hline Neoadjuvant chemotherapy & & & 0.58 \\
\hline Presence & 1 & 38 & \\
\hline Absence & 5 & 77 & \\
\hline Recurrence & & & 0.03 \\
\hline Presence & 3 & 18 & \\
\hline Absence & 3 & 97 & \\
\hline
\end{tabular}

For all P-values a $\chi^{2}$ test was performed. VATS, video-assisted thoracic surgery; pT, pathological depth of tumor invasion; pN, pathological lymph node stage.

A

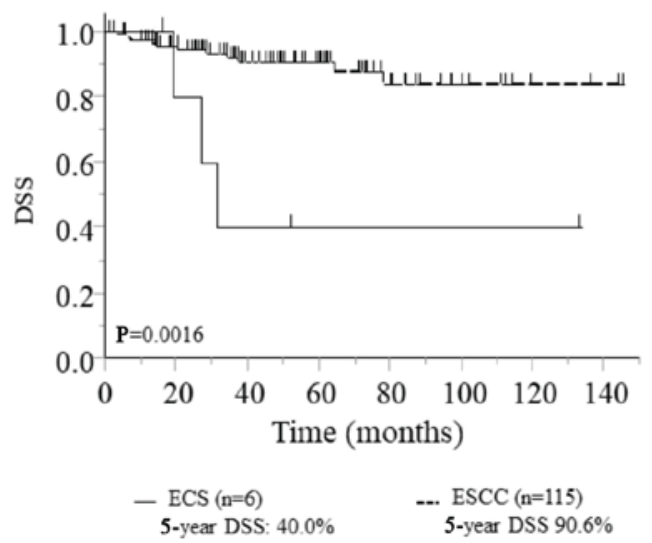

B

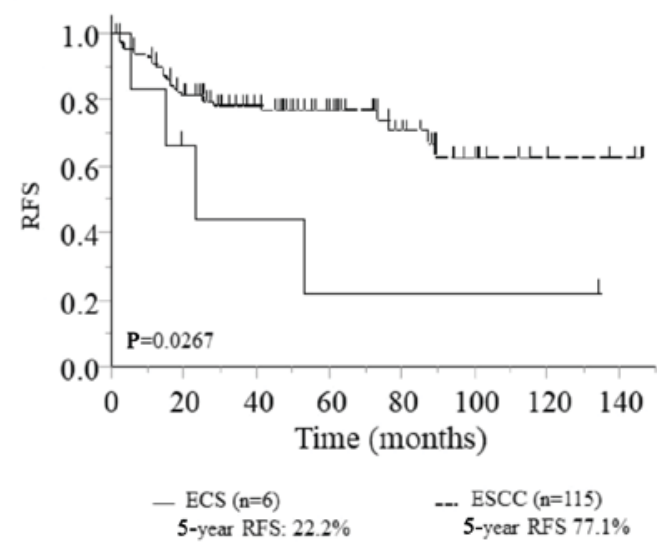

Figure 7. Associations between pathological features and DSS or RFS time. Pathological features consisted of ECS and ESCC with the same depth of tumor invasion. ECS was associated with significantly shorter (A) DSS and (B) RFS times compared with ESCC. DSS, disease-specific survival; RFS, relapse-free survival; ECS, esophageal carcinosarcoma; ESCC, esophageal squamous cell carcinoma.

anticancer drugs. It has been reported that recurrent tumors may be derived from sarcoma (37) with strong expression of ZEB1 being expected. For the treatment of recurrent tumors with expected anticancer drug resistance, no further promising results will be obtained unless molecular targeted treatment is applied. In addition, in a previous study in our 
laboratory (Department of Surgery, Kitasato University School of Medicine), it was confirmed that the suppression of ZEB1 expression in triple-negative breast cancer resulted in a loss of resistance to phenylbutyrate in the majority of cases (13).

Based on the aforementioned finding, the present study aimed to indicate the molecular uniqueness of ESC by confirming its specificity, as numerous molecules could be associated with EMT, including ZEB1, Snail 1 and Twist 1. According to the results of the comparative investigation, it was revealed that ZEB1 is highly characteristic of sarcoma components of EMT in ESC. However, a limitation of the present study is that it cannot fully explain the association between ZEB1 and poor prognosis. Despite that ZEB1 indicated an association with ESC, the present study did not examine this association on a molecular level, therefore further investigation is required.

In conclusion, a detailed clinicopathological analysis of ECS was performed in the present study, and its unique clinical and molecular features were identified. The features of ECS indicated in the present study may be of great assistance in further developing a novel therapeutic strategy for the drug-resistant neoplasm.

\section{Acknowledgements}

Not applicable.

\section{Funding}

No funding was received.

\section{Availability of data and materials}

All data generated or analyzed during the present study are included in this published article.

\section{Authors' contributions}

$\mathrm{HH}$ and KY conducted the conception and design, acquisition of data, analysis of data and drafting the manuscript. MWat performed the acquisition of data, and the drafting and revising of the manuscript. MWas, AE, MK and HMo aided with acquisition of data and assisted with revising the manuscript. YK aided with the statistical analysis and analysis of data. $\mathrm{KH}$ and HMi participated in the design and coordination of the study and assisted with revising the manuscript. All authors read and approved the final manuscript.

\section{Ethics approval and consent to participate}

The present study was conducted in accordance with the Declaration of Helsinki and approved by the Research Ethics Committee of Kitasato University School of Medicine (Sagamihara, Japan). Written informed consent was obtained from each patient.

\section{Patient consent for publication}

Written informed consent was obtained from each patient for the publication of their data.

\section{Competing interests}

The authors declare that they have no competing interests.

\section{References}

1. Virchow RL: Die Krankhalften Geschwulste. A Hirschwald, Berlin Volume 2: pp181-182, 1865.

2. Japan Esophageal Society: Japanese classification of esophageal cancer, 11th edition: Part II and III. Esophagus 14: 37-65, 2017.

3. Kashiwabara K, Sano T, Oyama T, Najahima T, Makita F, Hashimoto N, Iwanami K, Kawashima O, Matsumoto T and Matsuzaki Y: A case of esophageal sarcomatoid carcinoma with molecular evidence of a monoclonal origin. Pathol Res Pract 197: 41-46, 2001.

4. Handra-Luca A, Terris B, Couvelard A, Molas G, Degott C and Flejou JF: Spindle cell squamous carcinoma of the oesophagus: An analysis of 17 cases, with new immunohistochemical evidence for a clonal origin. Histopathology 39: 125-132, 2001.

5. Matsumoto T, Fujii H, Arakawa A, Yamasaki S, Sonoue H, Hattori K, Kajiyama Y, Hirose S and Tsurumaru M: Loss of heterozygosity analysis shows monoclonal evolution with frequent genetic progression and divergence in esophageal carcinosarcoma. Hum Pathol 35: 322-327, 2004.

6. Larue L and Bellacosa A: Epithelial-mesenchymal transition in development and cancer: Role of phosphatidylinositol 3' kinase/AKT pathways. Oncogene 24: 7443-7454, 2005.

7. Nieto MA: The ins and outs of the epithelial to mesenchymal transition in health and disease. Annu Rev Cell Dev Biol 27: 347-376, 2011.

8. Nakazawa T, Nobusawa S, Ikota H, Kuwano H, Takeyoshi I and Yokoo H: Wide expression of ZEB1 in sarcomatous component of spidle cell carcinoma of esophagus. Pathol Int 65: 635-643, 2015.

9. Brabletz S and Brabletz T: The ZEB/miR-200 feedback loop a motor of cellular plasticity in development and cancer? EMBO Rep 11: 670-677, 2010.

10. Lim YY, Wright JA, Attema JL, Gregory PA, Bert AG, Smith E, Thomas D, Lopez AF, Drew PA, Khew-Goodall Y and Goodall GJ: Epigenetic modulation of the miR-200 family is associated with transition to a breast cancer stem-cell-like state. J Cell Sci 126: 2256-2266, 2013.

11. Zhou X, Wang Y, Shan B, Han J, Zhu H, Lv Y, Fan X, Sang M, Liu XD and Liu W: The downregulation of miR200c/141 promotes ZEB1/2 expression and gastric cancer progression. Med Oncol 32: 428, 2015.

12. Sobin LH and Wittekind C: TNM classification of malignant tumors. 6th ed. New York: Wiley-Liss; 2002.

13. Kikuchi M, Yamashita K, Waraya M, Minatani N, Ushiku H, Kojo K, Ema A, Kosaka Y, Katoh H, Sengoku N, et al: Epigenetic regulation of ZEB1-RAB25/ESRP1 axis plays a critical role in phenylbutyrate treatment-resistant breast cancer. Oncotarget 7: 1741-1753, 2016.

14. Zhu DY, Guo QS, Li YL, Cui B, Guo J, Liu JX and Li P: Twist1 correlates with poor differentiation and progression in gastric adenocarcinoma via elevation of FGFR2 expression. World $\mathrm{J}$ Gastroenterol 20: 18306-18315, 2014.

15. Sung CO, Lee KW, Han S and Kim SH: Twist1 is up-regulated in gastric cancer-associated fibroblasts with poor clinical outcomes. Am J Pathol 179: 1827-1838, 2011.

16. Dong H, Xie L, Tang C, Chen S, Liu Q, Zhang Q, Zheng W, Zheng $\mathrm{Z}$ and Zhang H: Snaill correlates with patient outcomes in E-cadherin-preserved gastroesophageal junction adenocarcinoma. Clin Transi Oncol 16: 783-791, 2014.

17. Yu J, Li J, Chen Y, Cao W, Lu Y, Yang J and Xing E: Snail enhances glycolysis in the epithelial-mesenchymal transition process by targeting FBP1 in gastric cancer. Cell Physiol Biochem 43: 31-38, 2017.

18. Owens WD, Felts JA and Spitznagel EL Jr: ASA physical status classifications: A study of consistency of ratings. Anesthesiology 49: 239-243, 1978

19. Dindo D, Demartines N and Clavien PA: Classification of surgical complications: A new proposal with evaluation in a cohort of 6336 patients and results of a survey. Ann Surg 240: 205-213, 2004.

20. Kimura H, Konishi K, Kawamura T, Nojima N, Satou T, Maeda K, Yabushita K, Kuroda Y, Tsuji M and Miwa A: Esophageal sarcomas: Report of three cases. Dig Surg 16: 244-247, 1999. 
21. Ji F, Xu YM and Xu CF: Endoscopic polypectomy: A promising therapeutic choice for esophageal carcinosarcoma. World J Gastroenterol 15: 3448-3450, 2009.

22. Wang L, Lin Y, Long H, Liu H, Rao H, He Y, Rong $\mathrm{T}$ and Liang Y: Esophageal carcinosarcoma: A unique entity with better prognosis. Ann Surg Oncol 20: 997-1004, 2013.

23. Takemura M, Osugi H, Takeda N, Okuda E, Ueno $M$ and Kinoshita H: Three cases of resected so-called carcinosarcoma of the esophagus and review of the japanese literature. J Jpn Surg Assoc 62: 659-664, 2001 (In Japanese).

24. Sung CO, Choi H, Lee KW and Kim SH: Sarcomatoid carcinoma represents a complete phenotype with various pathways of epithelial mesenchymal transition. J Clin Pathol 66: 601-606, 2013.

25. Pang A, Carbini M, Moreira AL and Maki RG: Carcinosarcomas and related cancers: Tumors caught in the act of epithelial-mesenchymal transition. J Clin Oncol Dec 36: 210-216, 2018.

26. Viswanathan VS, Ryan MJ, Dhruv HD, Gill S, Eichhoff OM, Seashore-Ludlow B, Kaffenberger SD, Eaton JK, Shimada K, Aguirre AJ, et al: Dependency of a therapy-resistant state of cancer cells on a lipid peroxidase pathway. Nature 547: 453-457, 2017.

27. Horiguchi K, Sakamoto K, Koinuma D, Semba K, Inoue A, Inoue S, Fujii H, Yamaguchi A, Miyazawa K, Miyazono K and Saitoh M: TGF- $\beta$ drives epithelial-mesenchymal transition

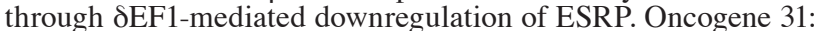
3190-3201, 2012

28. Joseph JV, Conroy S, Tomar T, Eggens-Meijer E, Bhat K, Copray S, Walenkamp AM, Boddeke E, Balasubramanyian V, Wagemakers M, et al: TGF- $\beta$ is an inducer of ZEB1-dependent mesenchymal transdifferentiation in glioblastoma that is associated with tumor invasion. Cell Death Dis 5: e1443, 2014.

29. Korpal M, Lee ES, Hu G and Kang Y: The miR-200 family inhibits epithelial-mesenchymal transition and cancer cell migration by direct targeting of E-cadherin transcriptional repressors ZEB1 and ZEB2. J Biol Chem 283: 14910-14914, 2008.
30. Gregory PA, Bert AG, Paterson EL, Barry SC, Tsykin A, Farshid G, Vadas MA, Khew-Goodall Y and Goodall GJ: The miR-200 family and miR-205 regulate epithelial to mesenchymal transition by targeting ZEB1 and SIP1. Nat Cell Biol 10: 593-601, 2008.

31. Park SM, Gaur AB, Lengyel E and Peter ME: The miR-200 family determines the epithelial phenotype of cancer cells by targeting the E-cadherin repressors ZEB1 and ZEB2. Gene Dev 22: 894-907, 2008.

32. Zhang B, Xiao Q, Yang D, Li X, Hu J, Wang Y and Wang W: Spindle cell carcinoma of the esophagus: A multicenter analysis in comparison with typical squamous cell carcinoma. Medicine (Baltimore) 95: e4768, 2016.

33. Aigner K, Dampier B, Descovich L, Mikula M, Sultan A, Schreiber M, Mikuliys W, Brabletz T, Strand D, Obrist P, et al: The transcription factor ZEB1 (deltaEF1) promotes tumour cell dedifferentiation by repressing master regulators of epithelial polarity. Oncogene 26: 6979-6988, 2007.

34. Arima Y, Hayashi H, Sasaki M, Hosonaga M, Goto T, Chiyoda T, Kuninaka S, Shibata T, Ohata H, Nakagama $\mathrm{H}$, et al: Induction of ZEB proteins by inactivation of RB protein is key determinant of mesenchymal phenotype of breast cancer. J Biol Chem 287: 7896-7906, 2012

35. Spaderna S, Schmalhofer O, Wahlbuhl M, Dimmler A, Bauer K, Sultan A, Hlubek F, Jung A, Strand D, Eger A, et al: The transcriptional repressor ZEB1 promotes metastasis and loss of cell polarity in cancer. Cancer Res 68: 537-544, 2008

36. Arumugam $\mathrm{T}$, Ramachandran V, Fournier KF, Wang $\mathrm{H}$, Marquis L, Abbruzzese JL, Gallick GE, Logsdon CD, McConkey DJ and Choi W: Epithelial to mesenchymal transition contributes to drug resistance in pancreatic cancer. Cancer Res 69: 5820-5828, 2009.

37. Sanada Y, Hihara J, Yoshida K and Yamaguchi Y: Esophageal carcinosarcoma with intramural metastasis. Dis Esophagus 19: 119-131, 2006. 